\title{
Incentivos tributarios para generación de energía a través de fuentes no convencionales en Colombia*
}

\section{Tax incentives for power generation through non-conventional sources in Colombia}

\author{
JAVIER ALEXÁNDER MORENO SUÁREZ ${ }^{1}$
}

\section{Sumario}

I. Introducción. II. Generación de energía. III.Aproximación al concepto de medioambiente desde lo jurídico. IV. Fiscalidad ambiental. V. Beneficios tributarios para la generación de energía. Bibliografía.

\section{Resumen}

La Ley 1715 de 2014 estableció una serie de incentivos de tipo tributario, contable y arancelario con el fin de promover el desarrollo de proyectos de generación a través de Fuentes No Convencionales de Energía Renovables (FNCER), los cuales fueron reglamentados en el Decreto 2143 de 2015, designando al Ministerio de Minas y Energía y a la Unidad de Planeación Minero Energética -UPME- para establecer los procedimientos para acceder a los beneficios; dichas entidades han expedido una serie de resoluciones con el fin de poder expedir las certificaciones necesarias que permitan usar los incentivos.

Palabras clave: Tributo Ambiental, Fiscalidad Ambiental, Incentivos Tributarios Colombia, Generación de Energía, Incentivos para la Generación de Energía, Fuentes No Convencionales de Energía Renovables, Fuentes No Renovables, Fuentes Convencionales, Deducciones en el Impuesto a la Renta, Depreciación Acelerada, Exclusión del IVA, Exención en el Gravamen Arancelario.

\section{Abstract}

Law 1715 of 2014 established a series of tax, accounting and tariff incentives in

${ }^{1}$ Estudiante de la Maestría en Derecho con Énfasis en Tributación, de la Universidad Externado de Colombia, Certificación en IFRS emitida por el ICAEW, Especialista en Contabilidad Financiera Internacional de la Universidad Javeriana, Especialista en Ciencias Tributarias de la Fundación Universidad Central, Contador Público de la Universidad Nacional.Correo-e: jams76@gmail.com 
order to promote the development of generation projects through Non-Conventional Renewable Energy Sources (FNCER), which were regulated in Decree 2143 of 2015, appointing the Ministry of Mines and Energy and the Energy Mining Planning Unit -UPME- to establish the procedures for accessing the benefits; these entities have issued a series of resolutions in order to be able to issue the necessary certifications that allow the use of the Incentives.

Keywords: Environmental Tribute, Environmental Taxation, Tax Incentives Colombia, Energy generation ,Incentives for Energy Generation, Non-Conventional Renewable, Energy Sources, Non-renewable Sources, Conventional Sources, Deductions from Income Tax, Accelerated Depreciation, Exemption from VAT, Exemption from Tariff Rate.

\section{Introducción}

El uso de los recursos naturales puede generar problemas de escasez, dada la relación inversa que existe entre la cantidad de bienes y servicios y la calidad del medioambiente. Así, el nuevo modelo de desarrollo basado en la internalización del concepto de desarrollo sustentable ha inducido fuertemente en el sector energético, donde es evidente la interacción con el medioambiente, así: "el tipo de desarrollo que hemos conocido desde la revolución industrial, basado en el saqueo del medio, ha tenido un efecto acumulado cada vez más visible y sus costes, individuales y sociales, son cada vez más evidentes" (Riechmann y otros, 1995).

En este contexto, el presente trabajo analiza incentivos creados por la Ley 1715 de 2014, con el fin de promover en Colombia la sustitución de la forma de producción de energías convencionales.

En este orden de ideas, el trabajo desarrolla conceptos relacionados con la fiscalidad ambiental, la obligación y mecanismos constitucionales que tiene el Gobierno para proteger el medioambiente, y da cuenta de la legislación que busca fomentar la generación de energías a través de Fuentes No Convencionales de Energía (FNCE).

\section{Generación de energía}

La energía eléctrica es un recurso indispensable para el desarrollo de muchas de las actividades que se deben realizar en el mundo actual, actividades que afectan todo tipo de industrias -salud, entretenimiento, transformación, transporte-, y esto se debe a las características que tiene, como son su fácil obtención a partir de las fuentes primarias y su fácil transmisión; de tal modo, la generación de electricidad es la transformación de diferentes fuentes de energía en electricidad.

\section{A. Fuentes No Convencionales de Energía (FNCE)}

Las Fuentes Convencionales son aquellos recursos de energía que son utilizados de manera intensiva; las Fuentes No Convencionales de Energía (FNCE), por su parte, son aquellos recursos de energía que son ambientalmente sostenibles, pero que no son utilizados o su uso es marginal (Sarmiento López, 2010).

Adicionalmente, ya en el ordenamiento jurídico colombiano encontramos que la Ley 1715 de 2014 incorpora una división más al considerar que existe una categoría denomina Fuentes No Convencionales de 
Energía Renovables (FNCER), las cuales nos corresponde desarrollar.

\section{Tipos de Fuentes No Convencionales} de Energía Renovables (FNCER): la delimitación de la presente lista se hace siguiendo la Ley 1715 de 2014, la cual define como FNCER la Biomasa, los pequeños aprovechamientos hidroeléctricos, la eólica, la geotérmica, la solar y los mares.

i. Energía Solar: la Energía Solar es aquella que proviene del Sol y es la principal fuente de energía del planeta Tierra; esta energía calienta la atmósfera, la superficie de los continentes y los mares, estimula el crecimiento de las plantas e influye en el clima. "La energía solar se transfiere a la superficie de la Tierra a través del espacio en cuantos de energía llamados fotones que interactúan con la atmósfera y la superficie terrestre, llegando a la tierra por dos vías diferentes: incidiendo en los objetos iluminados por el sol (radiación directa) y por reflexión de la radiación solar absorbida en la atmósfera y en el polvo atmosférico (radiación difusa)" (Isagén S.A. E.S.P., 2005).

ii. Energía hídrica: la energía hídrica o hidráulica, según la RAE, es la energía "producida por el movimiento del agua" (Real Academia de la Lengua Española). La energía hídrica es la más desarrollada en Colombia según la UPME, referenciada en el Plan Energético Nacional 2006-2025; la Ley 1715 de 2014 le da el estatus de FNCER porque incorpora el concepto aprovechamiento de los cuerpos de agua a pequeña escala, lo cual hace que se disminuya en gran medida el impacto negativo ambiental que tiene el uso de este tipo de energía.

iii. Energía eólica: la energía eólica es la energía del viento que se debe al movimiento del aire ocasionado por el desigual calentamiento de la superficie terrestre (Vega de Kuyper y Ramírez Morales, 2014).

La Ley 1715 de 2014 define la energía eólica como aquella obtenida de una FNCER que consiste en el movimiento de las masas de aire.

iv. Energía por biomasa: la Ley 1715 de 2014 define la energía de la biomasa como aquella obtenida a partir de una FNCER, que se basa en la degradación espontánea o inducida de cualquier tipo de materia orgánica que ha tenido su origen inmediato como consecuencia de un proceso biológico, y toda materia prima vegetal originada por el proceso de fotosíntesis, así como los procesos metabólicos de organismos heterótrofos ${ }^{2}$, y que no contienen o hayan estado en contacto con trazas de elementos que confieren algún grado de peligrosidad.

v. Energía geotérmica: la Ley 1715 de 2015 define la energía térmica como aquella energía obtenida de la FNCER que consiste en el calor que yace del subsuelo terrestre.

Según la UPME, "si bien Colombia no es uno de los países con mayor potencial para

\footnotetext{
2 "Heterótrofos: adj. Biol. Dicho de un organismo: Incapaz de elaborar su propia materia orgánica a partir de sustancias inorgánicas, por lo que debe nutrirse de otros seres vivos; p. ej., los animales y los hongos" (Real Academia de la Lengua Española).
} 
el aprovechamiento del recurso geotérmico, sí cuenta con zonas específicas, como lo son la zona volcánica del Nevado del Ruiz y la región de influencia de los volcanes Chiles, Cerro Negro y Azufral en la frontera con Ecuador, zonas en las que el recurso puede ser aprovechado para la generación de decenas de MW a muy bajos costos de producción y operación" (Unidad de Planeación Minero Energética (UPME), 2015).

\section{vi. Energía de los mares y los océanos: los} océanos son sistemas captadores y acumuladores de energía; el aprovechamiento térmico de esta energía presenta varias facetas, que van desde los gradientes ${ }^{3}$ térmicos y salinos hasta el movimiento del agua, el fenómeno de las mareas (Gárcia Galludo, Goded Velarde y Suárez Navarro, 2001); es decir, la energía del mar es la que tienen las olas, mareas, corrientes y gradientes de temperatura.

La Ley 1715 de 2014 la define como la obtenida a partir de aquella FNCER que comprende fenómenos naturales marinos, como son las olas, las corrientes marinas, los gradientes térmicos oceánicos y los gradientes de salinidad, entre otros.

En seguida se revisarán los otros tipos de fuentes de energía, aquellas que son limitadas.

\section{Fuentes de energía no renovables}

La RAE define las energías renovables como aquellas que se presentan de manera continua e inagotable en la naturaleza, con lo cual la expresión No Renovables hace referencia a las fuentes de energía que se obtienen o se encuentran en la naturaleza de forma limitada. Según Vega de Kuyper y Ramírez Morales, "suponen entorno al 80\% de la energía mundial y sobre ella se ha construido el inseguro modelo energético actual" (Vega de Kuyper y Ramírez Morales, 2014).

En Colombia, la Ley 1715 de 2014 no habla de Fuentes de Energía No Renovable, pero hace referencia a las fuentes convencionales de energía, y las define como aquellos recursos de energía que son utilizados de manera intensiva y ampliamente comercializados en el país.

En este sentido, por su potencial hidrológico y sus montañas, en Colombia la principal fuente de generación es la hidroeléctrica, seguida de la termoeléctrica (gas, diésel y carbón); contrastado lo anterior con la definición de la Ley 1715 de 2014, la energía hidráulica y térmica son las fuentes convencionales, lo cual se puede validar con la siguiente tabla tomada de los informes anuales de $\mathrm{XM}^{4}$, que contiene la Capacidad Efectiva Neta $(\mathrm{CEN})^{5} \mathrm{del}$ Sistema Interconectado Nacional (SIN). A continuación se presentan las siguientes gráficas.

\footnotetext{
3 "Razón entre la variación del valor de una magnitud en dos puntos próximos y la distancia que los separa" (Real Academia de la Lengua Española).

${ }^{4} \mathrm{XM}$ es filial de ISA especializada en la Gestión de Sistemas de Tiempo Real, es el encargado de la operación del Sistema Interconectado Nacional (SIN) colombiano y la Administración del Mercado de Energía en Colombia, incluyendo las transacciones internacionales de electricidad con Ecuador (www.xm.com.co).

${ }^{5}$ Capacidad Efectiva Neta (CEN): "Es la máxima capacidad de potencia neta (expresada en valor entero en MW) que puede suministrar una planta y/o unidad de generación en condiciones normales de operación, medida en la frontera comercial. Se calcula como la Capacidad Nominal menos el Consumo Propio de la planta y/o unidad de generación (Comisión de Regulación de Energía y Gas).
} 


\begin{tabular}{|c|c|c|c|c|}
\hline Recursos & $\begin{array}{l}2015 \\
(\mathrm{MW})\end{array}$ & $\begin{array}{l}2016 \\
(\mathrm{MW})\end{array}$ & $\begin{array}{c}\text { Participación } \\
(\%)\end{array}$ & $\begin{array}{c}\text { Variación } \\
(\%)\end{array}$ \\
\hline Hidráulicos & 10,892 & 10,963 & $66,06 \%$ & $0.65 \%$ \\
\hline Térmicos & 4,743 & 4,728 & $28,49 \%$ & $-0.32 \%$ \\
\hline Gas & 1,548 & 2,128 & & $37.47 \%$ \\
\hline Carbón & 1,339 & 1,329 & & $-0.75 \%$ \\
\hline Fuel - Oil & -- & -- & & $0.00 \%$ \\
\hline Combustóleo & 299 & 187 & & $-37.46 \%$ \\
\hline ACPM & 1,247 & 774 & & $-37.93 \%$ \\
\hline Jet1 & 46 & 46 & & $0.00 \%$ \\
\hline Gas-Jet A1 & 264 & 264 & & $0.00 \%$ \\
\hline Menores & 698.42 & 771.52 & $4,65 \%$ & $10 \%$ \\
\hline Hidráulicos & 608.55 & 648.10 & & $6.50 \%$ \\
\hline Térmicos & 71.45 & 105 & & $46.96 \%$ \\
\hline Eólica & 18.42 & 18.42 & & $0.00 \%$ \\
\hline Cogeneradores & 86.60 & 99.60 & $0,60 \%$ & $15.01 \%$ \\
\hline Autogeneradores & -- & 32 & $0,20 \%$ & $100 \%$ \\
\hline Total SIN & $16,420.02$ & $16,594.52$ & $100 \%$ & $1.06 \%$ \\
\hline
\end{tabular}

Fuente: XM, Informes anuales.

Gráfica 1. Capacidad efectiva neta (CEN) del SIN a 31 de diciembre de 2016

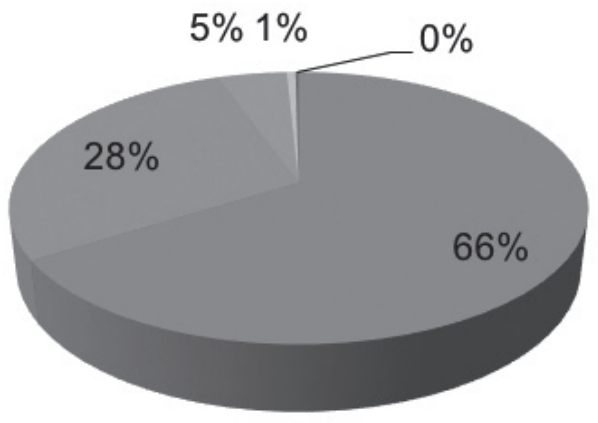

"Hidráulicos

-Térmicos

Menores

Cogeneradores

- Autogeneradores 
De la información publicada por $\mathrm{XM}^{6}$, se concluye que el SIN creció un 1,06\% entre 2015 y 2016, lo que, traducido en $\mathrm{MW}^{7}$, son 174.5; adicionalmente, la alta dependencia que tiene el SIN de dos fuentes de generación, la hidráulica y la térmica, la CEN instalada que tiene el SIN al cierre de 2016, es de 16.594 MW, de cuyo total el 66\% corresponde a la generación hidráulica que representa 10.963 MW y el 28,49\% a generación térmica (combustibles fósiles) que son 4.728 MW. Estas dos fuentes de generación de energía eléctrica representan alrededor del 95\%, es decir, que generan 15.691 MW, mientras las plantas menores, cogeneradores y autogeneradores $^{8}$ llegan solamente al $5 \%$.

\section{Aproximación a un concepto de medioambiente desde lo jurídico}

\section{La Corte Constitucional ha definido el} medioambiente como un derecho ${ }^{9}$, de la siguiente forma:

El ambiente sano, como se ha mencionado forma parte de los derechos colectivos cuya esencia transciende el concepto de derecho individual para radicarse en el ser social, el cual incumbe, además, a cada una de las esferas que componen el entramado social, de manera que su conservación impone deberes correlativos a los particulares, la sociedad, las empresas, al Estado y la comunidad internacional.

Se acepta al medioambiente sano como un derecho del cual son titulares todas las personas, quienes a su vez se encuentran legitimadas para participar en las decisiones que puedan afectarlo y deben colaborar en su conservación. También como un deber que se le impone a todos y particularmente al Estado: i) proteger su diversidad e integridad, ii) salvaguardar las riquezas naturales de la Nación, iii) conservar las áreas de especial importancia ecológica, iv) fomentar la educación ambiental, v) planificar el manejo y aprovechamiento de los recursos naturales para así garantizar su desarrollo sostenible, su conservación, restauración o sustitución, vi) prevenir y controlar los factores de deterioro ambiental, vii) imponer las sanciones legales y exigir la reparación de los daños causados al ambiente; $y$ viii) cooperar con otras $\mathrm{Na}$ ciones en la protección de los ecosistemas situados en las zonas de frontera" (Sentencia C-595 de 2010).

El derecho a un medioambiente sano, como bien jurídico constitucional, es la base de la tributación ambiental, pues el hecho de

\footnotetext{
${ }^{6}$ La información contenida en la tabla base para para la elaboración de la gráfica puede ser consultada en http://informesanuales.xm.com.co/2016/SitePages/operacion/2-6-Capacidad-efectivaneta.aspx

${ }^{7} \mathrm{MW}$, el vatio es representado por $\mathrm{W}$, por lo cual MW es una unidad de medida que representa a un millón de vatios $1 \mathrm{mw}=1.000 .000 \mathrm{w}$.

${ }^{8}$ La Ley 1715 de 2014 define la Cogeneración como "producción combinada de energía eléctrica y energía térmica que hace parte integrante de una actividad productiva", y la Autogeneración, como "Aquella actividad realizada por personas naturales o jurídicas que producen energía eléctrica principalmente, para atender sus propias necesidades".

${ }^{9}$ La Constitución, al consagrar el ambiente sano como un derecho, está haciendo la incorporación de los derechos de Tercera Generación, concepto que será desarrollado más adelante.
} 
que esté reconocido en sede constitucional hace que todo el ordenamiento se informe de la necesidad de proteger el medioambiente por intermedio del uso de los instrumentos que sean necesarios (Betancor y otros, 2002).

La siguiente lista contiene algunos artículos de la Constitución Política de Colombia que permiten identificar los deberes del Gobierno para proteger y promover conductas amigables con el medioambiente, y además se establece la justificación para que el Gobierno intervenga en su protección, ya sea a través de una regulación directa o indirecta de mecanismo de intervención al mercado.

- Derecho a un medioambiente sano, Artículo 79.

- Saneamiento ambiental, Artículo 49.

- Función ecológica de la propiedad, Artículo 58.

- Bienes de uso público, Artículo 63.

- Desarrollo sostenible, Artículo 80.

- El ambiente como límite a la libertad económica, Artículo 333.

- Intervención del Estado en la Economía para la preservación de un ambiente sano, Artículo 334.

Teniendo en cuenta las anteriores referencias de la Constitución Nacional, se puede afirmar que la Constitución de Colombia de 1991 es ecológica; en este sentido lo ha expresado la Corte Constitucional, mediante la Sentencia C-126 de 1998:
La Constitución de 1991 modificó profundamente la relación normativa de la sociedad colombiana con la naturaleza. Por ello esta Corporación ha señalado, en anteriores decisiones, que la protección del medioambiente ocupa un lugar tan trascendental en el ordenamiento jurídico que la Carta contiene una verdadera "constitución ecológica", conformada por todas aquellas disposiciones que regulan la relación de la sociedad con la naturaleza y que buscan proteger el medioambiente. Igualmente la Corte ha precisado que esta Constitución ecológica tiene dentro del ordenamiento colombiano una triple dimensión: de un lado, la protección al medioambiente es un principio que irradia todo el orden jurídico puesto que es obligación del Estado proteger las riquezas naturales de la Nación. De otro lado, aparece como el derecho de todas las personas a gozar de un ambiente sano, derecho constitucional que es exigible por diversas vías judiciales. Y, finalmente, de la constitución ecológica derivan un conjunto de obligaciones impuestas a las autoridades y a los particulares. Es más, en varias oportunidades, la Corte ha insistido en que la importancia del medioambiente en la Constitución es tal que implica para el Estado, en materia ecológica, "unos deberes calificados de protección”. (Sentencia C-126 de 1998).

Teniendo claro lo comentado, a continuación se seguirá profundizando en el estudio.

\section{Fiscalidad ambiental}

A efectos metodológicos, a lo largo del presente escrito los términos impuestos ambientales, tributos ambientales, gravámenes ambientales, impuestos ecológicos, impues- 
tos verdes y ecotasas se usan como sinónimos y de forma indistinta.

El término tributo encierra diferentes conceptos, por ejemplo, los de impuestos y tasas se deben diferenciar para ampliar el análisis y esto se debe a la naturaleza fiscal de las figuras utilizadas; las tasas financian el costo de un determinado servicio público, mientras que los impuestos carecen de una finalidad financiera directa, por lo cual, si la actuación fiscal no tiene una destinación específica, así todos los recursos recaudados sean destinados a temas ambientales, se trata de un impuesto.

Otro concepto que se debe definir es la contribución, la cual se define como una compensación pagada con carácter obligatoria a un ente público, con ocasión de una obra realizada por él con fines de utilidad pública pero que proporciona ventajas especiales a los particulares

Las diferencias existentes entre los tres conceptos de tributos enunciados son de carácter técnico, lo que puede justificar su uso bajo la denominación de imposición ambiental. Con esta perspectiva simplificada el Tributo Ambiental como "un pago obligatorio que deben realizar los agentes que emiten sustancias contaminantes (a partir o no de un determinado nivel mínimo), siendo calculado por la aplicación de un tipo impositivo (fijo o variable) a una base imponible relacionada con el nivel de descargas al medio natural" (Gago Rodríguez y Labandeira Villot, 1999).

Dentro de la definición se evita cualquier referencia al uso de la recaudación con fines ambientales, en tanto no es condición necesaria para clasificar un tributo como ambiental por el solo hecho de destinar su recaudo a mejorar el medioambiente, y si se usara ese criterio cualquier tributo puede ser potencialmente ambiental.

Los tributos ambientales "son las prestaciones pecuniarias exigidas por un ente público con la finalidad principal de producir efectos de conservación, reparación, mejora $\mathrm{y}$, en general protección del medio ambiente" (Bokobo Moiche, 2000); en otras palabras, la definición de tributo ambiental sería aquel que mide y grava directamente las descargas contaminantes al medioambiente, aunque en la práctica pueden ser muchas las figuras impositivas que también pueden utilizarse para reducir el deterioro ambiental, como por ejemplo los impuestos aplicables al empleo de bienes cuya utilización es nociva para el medioambiente o las tasas que exigen según las emisiones contaminantes del contribuyente.

\section{Incentivos tributarios para la generación de energía}

Los incentivos tributarios son instrumentos económicos que contemplan una excepción a la aplicación de la regla tributaria ordinaria. Con este mecanismo, el Estado, en su facultad recaudatoria, deja de recibir algunos recursos, con el fin de producir o promocionar ciertas conductas o comportamientos que deben ser favorables con el medioambiente: "los incentivos fiscales ambientales son tratamientos excepcionales, en el sentido de que constituyen una excepción respecto de los tratamientos ordinarios. Por medio de ellos la administración tributaria deja de percibir determinadas cantidades para promocionar determinadas conductas 
ecológicas o favorables con el medioambiente. En este sentido pueden ejercer una influencia considerable sobre los hábitos de consumo y de comportamiento" (Bokobo Moiche, 2000).

El marco regulatorio de los incentivos para la generación de energía a través de fuentes no convencionales en Colombia es la Ley 1715 de 2014, cuyo objeto es promover el desarrollo y la utilización de las FNCE y en especial las Renovables (FNCER), a través de la Integración al Sistema Interconectado Nacional (SIN), el empleo en Zonas No Interconectadas (ZNI), la reducción de gases de efecto invernadero y la seguridad de abastecimiento energético; como objeto complementario tiene que promover la gestión eficiente de la energía, tanto en la eficiencia energética como en la respuesta a la demanda (Congreso de la República).

La finalidad de la ley es establecer un marco legal y los instrumentos para el aprovechamiento de las FNCER, así como fomentar la inversión, investigación y desarrollo de las tecnologías limpias para la generación de energía, para la eficiencia energética y para la respuesta a la demanda.

Adicionalmente, la Ley 1715 de 2014, en el artículo 4, declara como un asunto de utilidad pública e interés social, público y de conveniencia nacional, fundamental para asegurar la diversificación del abastecimiento energético pleno y oportuno, la competitividad de la economía colombiana, la protección del ambiente, el uso eficiente de la energía y la preservación y conservación de los recursos naturales renovables a través del empleo de las FNCER, y al respecto señala:
Esta calificación de utilidad pública o interés social tendrá los efectos oportunos para su primacía en todo lo referente a ordenamiento del territorio, urbanismo, planificación ambiental, fomento económico, valoración positiva en los procedimientos administrativos de concurrencia y selección, así como a efectos de expropiación forzosa.

Dicha ley consagra beneficios fiscales relacionados con el impuesto a la renta, el impuesto sobre las ventas y el gravamen arancelario, los cuales son definidos como incentivos a la inversión en proyectos de FNCE.

Para acceder a los beneficios de carácter fiscal, se debe revisar algunas definiciones contenidas en la Ley 1715 de 2014, y las adicionadas por el Decreto 2143 de 2015, las cuales tiene un carácter complementario (Artículo 2.2.3.8.1.1. del Decreto 1073 de 2015, Decreto Único Reglamentario del Sector de Minas y Energía).

Las definiciones del Decreto 2143 de 2015 son:

- Generadores de energía a partir de FNCE: son aquellos contribuyentes declarantes del Impuesto a la Renta y Complementarios y obligados a llevar contabilidad que generaren energía a través de FNCE.

- Nuevas inversiones en proyectos de FNCE: se considera como nuevas inversiones el aporte y/o erogaciones de recursos financieros que tengan como objetivo el desarrollo de FNCE, que se hagan a partir de la entrada en vigencia del Decreto 2149 de 2015, es decir, a partir del 4 de noviembre de 2015. 
- Nuevos proyectos de FNCE: son aquellas actividades interrelacionadas que se desarrollan de manera coordinada para instalar capacidad de generación de energía eléctrica a partir de FNCE desde la expedición del presente decreto. Estas actividades pueden incluir actividades como investigación y desarrollo tecnológico o, formulación e investigación preliminar, estudios técnicos, financieros, económicos y ambientales definitivos, adquisición de equipos, elementos, maquinaria, y montaje y puesta en operación.

- Etapas de proyectos de FNCE o gestión eficiente de la energía, para poder acceder a los beneficios de la ley, se entiende como etapas de los proyectos las siguientes:

$\checkmark$ Etapa de Preinversión, corresponde a la investigación y desarrollo.

$\checkmark$ Etapa de Inversión, son los estudios técnicos, financieros, económicos y ambientales definitivos, montaje e inicio de operación.

$\checkmark$ Etapa de Operación, corresponde a la administración, operación y mantenimiento.

Considerando lo anterior, se señalaran los incentivos tributarios que se establecieron en la mencionada ley.

\section{A. Impuesto sobre la Renta}

1. Deducción Especial: el artículo 11 de la Ley 1715 del 2014, como fomento a la investigación, desarrollo e inversión en el ámbito de la producción y utilización de energía a partir de FNCE, que los declarantes del Impuesto a la Renta pueden deducir anualmente el 50\% del total de la inversión realizada en los 5 años siguientes al año en que se ejecutó la inversión.

Este artículo fue reglamentado por el Decreto 2143 de 2015, indicando que la deducción especial del artículo 11 aplica para los contribuyentes del Impuesto a la Renta que realicen directamente nuevas erogaciones en investigación, desarrollo e inversión en el ámbito de la producción y utilización de energía a partir de FNCE; consiste en que al declarante le da el derecho a deducir hasta el $50 \%$ del valor de las inversiones.

Para acceder a este beneficio quien esté interesado debe obtener previamente la certificación del beneficio ambiental que expide el Ministerio de Ambiente y Desarrollo Sostenibles en los términos del artículo 158-2 del E.T. (hoy artículo $255 \mathrm{del} \mathrm{E.T.} .^{10}$ ), con las reglamentaciones que se expidan para tal fin.

El actual artículo 255 del E.T. es el siguiente:

Art. 255. Descuento para inversiones realizadas en control, conservación y mejoramiento del medio ambiente.

*-Adicionado-Las personas jurídicas que realicen directamente inversiones en control,

${ }^{10}$ La ley 1715 hace referencia directa al artículo 158-2 del E.T., pero la Ley 1819 de 2016 en su “Articulo 376 Vigencias y Derogatorias" deroga explícitamente el artículo 158-2 del E.T. y adicionalmente modifica el artículo 255 en cuyo parágrafo indica que la referencia que hace la Ley 1715 de 2014 al artículo 158-2 se entiende dirigida al artículo 255 del E.T. 
conservación y mejoramiento del medio ambiente, tendrán derecho a descontar de su impuesto sobre la renta a cargo el $25 \%$ de las inversiones que hayan realizado en el respectivo año gravable, previa acreditación que efectúe la autoridad ambiental respectiva, en la cual deberá tenerse en cuenta los beneficios ambientales directos asociados a dichas inversiones. No darán derecho a descuento las inversiones realizadas por mandato de una autoridad ambiental para mitigar el impacto ambiental producido por la obra o actividad objeto de una licencia ambiental.

PARÁGRAFO. El reglamento aplicable al artículo 158-2 del Estatuto Tributario antes de la entrada en vigencia de la presente ley, será aplicable a este artículo y la remisión contenida en la Ley 1715 de 2014 al artículo 158-2 del Estatuto Tributario, se entenderá hecha al presente artículo.

La deducción especial puede ser utilizada en un periodo no mayor de 5 años contados a partir del siguiente año en el cual se haga la nueva erogación; esta deducción puede ser hasta del 50\% de la nueva erogación y está limitada al 50\% de la Renta Líquida Gravable del Contribuyente antes de restar la deducción aplicando el artículo 177-1 del E.T.

Cuando la inversión sea realizada a través de contratos leasing financiero ${ }^{11}$ con opción irrevocable de compras, aplicará el beneficio tributario a partir del año siguiente en el cual se suscriba el contrato, siempre y cuando el locatario ejerza la opción de compra; si no se ejerce la opción los valores objeto del beneficio deben ser declarados como recuperación de deducciones conforme a los artículos 195 y 196 del E.T. en el año que no se decida ejercer la opción.

Para determinar la base de cálculo del beneficio de la deducción especial, se debe utilizar el artículo 127-1 del E.T.; este artículo fue modificado por la Ley 1819 de 2016, y en el literal b. del numeral 2) define el tratamiento del leasing financiero para el arrendatario:

\section{b. Para el arrendatario:}

i. Al inicio del contrato, el arrendatario deberá reconocer un activo y un pasivo por

\footnotetext{
${ }^{11}$ La definición de leasing financiero se encuentra en el literal a. del numeral 1) contenido en el artículo 127-1 del e.t. "a. Arrendamiento financiero o leasing financiero: Es aquel contrato, que tiene por objeto la adquisición financiada de un activo y puede reunir una o varias de las siguientes características:

"i. Al final del contrato se trasfiere la propiedad del activo al arrendatario o locatario.

"ii. El arrendatario o locatario tiene la opción de comprar el activo a un precio que sea suficientemente inferior a su valor comercial en el momento en que la opción de compra sea ejercida, de modo que al inicio del arrendamiento se prevea con razonable certeza que tal opción podrá ser ejercida. "iii. El plazo del arrendamiento cubre la mayor parte de la vida económica del activo incluso si la propiedad no se trasfiere al final de la operación.

“iv. Al inicio del arrendamiento el valor presente de los pagos mínimos por el arrendamiento es al menos equivalente al valor comercial del activo objeto del contrato. La dian podrá evaluar la esencia económica del contrato para comprobar si corresponde o no a una compra financiada.

"v. Los activos arrendados son de una naturaleza tan especializada que solo el arrendatario puede usarlos sin realizar en ellos modificaciones importantes. Los contratos de leasing internacional se someten a las reglas anteriores".
} 
arrendamiento, que corresponde al valor presente de los cánones de arrendamientos, la opción de compra y el valor residual de garantía en caso de ser aplicable, calculado a la fecha de iniciación del contrato, y a la tasa pactada en el contrato. La suma registrada como pasivo por el arrendatario, debe coincidir con la registrada por el arrendador como activo por arrendamiento.

Adicionalmente y de manera discriminada, se podrán adicionar los costos en los que se incurra para poner en marcha o utilización el activo siempre que los mismos no hayan sido financiados.

ii. El valor registrado en el activo por el arrendatario, salvo la parte que corresponda al impuesto a las ventas que vaya a ser descontado o deducido, tendrá la naturaleza de activo el cual podrá ser amortizado o depreciado en los términos previstos en este estatuto como si el bien arrendado fuera de su propiedad.

iii. El IVA pagado en la operación solo será descontable o deducible según el tipo de bien objeto del contrato por parte del arrendatario, según las reglas previstas en este estatuto.

iv. Cuando el arrendamiento financiero comprenda bienes inmuebles, la parte correspondiente a terrenos no será depreciable ni amortizable.

v. Los cánones de arrendamiento causados a cargo del arrendatario, deberán descomponerse en la parte que corresponda a abono a capital y la parte que corresponda a intereses o costo financiero. La parte correspondiente a abonos de capital, se cargará directamente contra el pasivo registrado por el arrendatario, como un menor valor de éste. La parte de cada canon correspondiente a intereses o costo financiero, será un gasto deducible para el arrendatario sometido a las limitaciones para la deducción de intereses.

vi.Al momento de ejercer la opción de compra, el valor pactado para tal fin se cargará contra el pasivo del arrendatario, debiendo quedar éste en ceros. Cualquier diferencia se ajustará como ingreso o gasto.

vii. En el evento en que el arrendatario no ejerza la opción de compra, se efectuarán los ajustes fiscales en el activo y en el pasivo, y cualquier diferencia que surja no tendrá efecto en el impuesto sobre la renta, siempre y cuando no haya generado un costo o gasto deducible, en tal caso se tratara como una recuperación de deducciones.

Cuando se realice enajenación de activos incluidos en los proyectos para el desarrollo de FNCE, antes de terminar el periodo de depreciación o amortización, deberán restituir las sumas resultantes de la aplicación de los beneficios como una renta por recuperación de deducciones conforme a los artículos 195 y 196 del E.T. en el año gravable en que se realice la enajenación.

Los beneficios fiscales no serán aplicables cuando las inversiones se efectúen a través de contratos de retroarriendo o lease back, o cualquier otra modalidad que no implique la transferencia de propiedad de los activos a su finalización.

2. Depreciación acelerada: el artículo 14 Ley 1715 del 2014 indica que se podrá realizar depreciación acelerada a las maquinarias, 
equipos y obras civiles para la preinversión, inversión y operación de las FNCE. Solo para este fin, la tasa anual de depreciación no podrá ser mayor a veinte por ciento $(20 \%)$; de requerirse variación, se debe comunicar a la DIAN.

A este beneficio pueden acceder los generadores de energía, a partir de FNCE que realicen nuevas inversiones en maquinaria, equipos y obras civiles adquiridos y/o construidos con posterioridad de la vigencia de la Ley 1715 de 2014.

Adicionalmente, se debe cumplir con el mismo requisito que tiene la deducción especial, es decir, obtener con anterioridad la certificación del artículo 158-2 del E.T. (hoy artículo 255 del E.T.).

\section{Requisitos para acceder a los benefi- cios en materia del Impuesto a la Renta:} conforme al artículo 11 de la Ley 1715 de 2014 , los proyectos que quieran aplicar a la deducción especial y a la depreciación acelerada por generación a través de FNCE, deberán obtener la Certificación de Incentivo Ambiental que expide el Ministerio de Ambiente y Desarrollo Sostenible a través de la ANLA, en los términos del artículo 158-2 del E.T. (hoy artículo 255 del E.T.).

Así mismo, los contribuyentes del Impuesto a la Renta, conforme al Decreto 2143 de 2015, deben obtener la certificación expedida por la UPME, en la cual se avala el proyecto de FNCE, los equipos, elementos y maquinaria, nacionales o importados, o la adquisición de servicios.

El desarrollo de la obtención de certificaciones de la ANLA y la UPME se desarrollará de manera conjunta, es decir, se abordarán impuesto a la Renta, Impuesto a las ventas y Gravamen Arancelario en un solo numeral.

Conforme a lo enunciado por la Ley 1715 de 2014, y tratado en párrafos anteriores, hay dos beneficios tributarios dirigidos a los contribuyentes del Impuesto a la Renta que hagan nuevas inversiones en proyectos de generación a través de FNCE, pero en el evento en que el contribuyente solicitante presenta pérdidas fiscales y está tributando por el sistema de renta presuntiva, ¿puede o no puede el contribuyente aplicar los beneficios? Y en caso de poder aplicarlos, ¿cómo puede aplicar dichos beneficios?

De acuerdo con la ley, los beneficios de la deducción especial y la depreciación acelerada de activos (art. 11 y 14 de la Ley 1715 de 2014) no son excluyentes, en la medida en que la ley permite aplicar los beneficios de forma conjunta, pero para dar respuesta se revisan de forma separada.

En primera instancia, la deducción especial contenida en el artículo 11, como se dijo en párrafos anteriores, consiste en tomar una deducción especial equivalente el $50 \%$ del valor de la inversión en proyectos de FNCE, pero este mismo artículo le impone una limitación a la aplicación de la deducción, la cual es que la deducción no podrá ser superior al 50\% de la renta líquida antes de restar el valor de la inversión, con lo cual, en el caso que presente pérdida fiscal antes de aplicar la deducción especial, no es factible tomar el beneficio por lo menos en ese año fiscal.

Ahora bien, la ley le permite al contribuyente aplicar este beneficio anualmente en la renta, por los 5 años siguientes al año grava- 
ble en que realizó la inversión, con lo cual si en el primer año no pudo aplicar la deducción puede aplicarla en los 4 años siguientes o restante, siempre y cuando no se supere el límite del $50 \%$ de la renta líquida del año en que se incluye la deducción, en otras palabras, en cualquiera de los 5 años siguientes se puede incluir total o parcialmente el beneficio siempre y cuando se tenga en cuenta la limitación.

Si durante este plazo de 5 años en los que se puede aplicar el beneficio, se presenten pérdidas fiscales recurrentes o la renta líquida no permita tomar dicho beneficio, el contribuyente solicitante lo pierde total o parcialmente.

En cuanto a la depreciación acelerada de activos (art. 14 de la Ley 1715 de 2014), la ley y el decreto reglamentario no imponen ninguna limitación ni tope en su aplicación, excepto que la cuota anual global de depreciación no puede ser superior al $20 \%$ del valor de la inversión, con lo cual si el contribuyente solicitante presenta pérdida fiscal y decide tomar este beneficio, lo que está haciendo en la práctica es incrementando la pérdida fiscal.

En el caso de que el contribuyente de renta tenga la intención de tomar los dos beneficios (deducción especial y depreciación acelerada), la situación que se puede presentar es la siguiente: como ya se dijo, la depreciación acelerada no tiene restricción cuantitativa ni temporal, con lo cual, si el contribuyente acelera la deprecación tomando una alícuota del $20 \%$ anual, puede llevar la renta líquida a una pérdida fiscal, con lo cual la aplicación de la deducción especial se debe postergar; si se aplica de forma consistente la alícuota del $20 \%$ la inversión objeto de la depreciación acelerada se terminará de depreciar en
5 años, que es mismo plazo que tiene el contribuyente para tomar la deducción especial. Para este tipo de situaciones, lo que se debe hacer es un análisis para determinar en primera instancia si se pueden aplicar los dos beneficios en la misma declaración o cuál es la combinación que optimiza la decisión, dándole prioridad a la deducción especial por la restricción temporal.

\section{B. Impuesto sobre las ventas}

El artículo 12 de la Ley 1715 de 2014 indica que los equipos, elementos, maquinaria y servicios nacionales o importados para la preinversión, inversión, producción y utilización de FNCE estarán excluidos de IVA, pero al igual que el incentivo en el Impuesto a la Renta el Ministerio de Ambiente y Desarrollo Sostenible certificará los equipos y servicios de acuerdo con un listado.

Para acceder al beneficio será suficiente la certificación de la Autoridad Nacional de Licencias Ambientales en la que se incluirán las cantidades y subpartidas arancelarias para soportar en la declaración de importación ante la DIAN la exclusión del IVA, como para solicitar la exclusión del IVA en las adquisiciones nacionales. En los dos casos se deberá obtener previamente la certificación expedida por el Ministerio de Minas y Energía, a través de la Unidad de Planeación Minero Energética (UPME).

Conforme al Decreto 2149 de 2015, previa la importación de los bienes o las adquisiciones nacionales, se deberá obtener la certificación expedida por la UPME, en la cual la entidad avalará el proyecto de FNCE y los equipos, elementos y maquinaria, nacionales o importados, o la adquisición de servicios. 
Adicionalmente, se deberá obtener la certificación emitida por la Autoridad Nacional de Licencias Ambientales de equipos y servicios excluidos del impuesto, para lo cual se basará en el listado elaborado por la UPME, y sus respectivas actualizaciones.

\section{Gravamen arancelario}

El artículo 13 Ley 1715 de 2014 indica que los titulares de inversiones en proyectos de FNCE contarán con exención del pago de los derechos arancelarios de importación para los casos de maquinaria, equipos, materiales e insumos, exclusivos para preinversión e inversión. Solo será aplicable si el único método de adquisición es la importación. Para obtener este incentivo, debe solicitarse con 15 días antes de la importación a la DIAN.

Los titulares de las nuevas inversiones, una vez expedidas las certificaciones de la UPME y de la Autoridad Nacional Ambiental de Licencias Ambientales, deberán remitir a la ventanilla Única de Comercio Exterior (VUCE) la solicitud de licencia previa, anexando las certificaciones.

Este beneficio se aplica de forma exclusiva para la maquinaria, equipos, materiales e insumos destinados únicamente a las etapas de preinversión e inversión. El Decreto 2149 de 2015 define los requisitos y son similares a los establecidos para la exclusión del IVA.

\section{Marco normativo que establece los requisitos para acceder beneficios tributarios}

Tanto la Ley 1715 de 2014, como el Decreto Reglamentario 2143 de 2015 exigen como requisitos para acceder a los benefi- cios, obtener previamente la certificación de incentivo ambiental que expide el Ministerio de Ambiente y Desarrollo Sostenible a través de la Autoridad Nacional de Licencias Ambientales (ANLA) y la certificación expedida por la UPME, en la cual se avalan el proyecto de FNCE, los equipos elementos y maquinaria, nacionales o importados, o la adquisición de servicios.

La UPMEN, a través de la Resolución 045 del 3 de febrero de 2016, establece los requisitos para solicitar la certificación que avala la documentación, con el fin de iniciar el trámite de certificación de beneficio ambiental ante la ANLA, para la exclusión del IVA y la Exención del Gravamen Arancelario, según los artículos 12 y 13 de la ley 1715 de 2014.

Para tal fin, la resolución establece que la UPME debe publicar a través de su página web la lista de equipos de bienes y servicios excluidos de IVA y Gravamen Arancelario, y define el procedimiento que debe seguir ya sea una personas natural o jurídica para actualizar y/o modificar dicha lista (Unidad de Planeación Minero Energética, 2016).

En el artículo 8 de la Resolución 045 de 2016 de la UPME, en concordancia con el Decreto 2143 de 2015 , se indica que quien esté interesado en solicitar la certificación para obtener los beneficios de los artículos 12 y 13 de la Ley 1715 de 2014, primero debe estar inscrito en el registro de proyectos de generación de energía eléctrica de la UPME, lo cual está regulado por la Resolución 0520 de 9 de octubre de 2007 -Por medio de la cual se establece el registro de proyectos de generación con el cual deben ser registrados los proyectos de generación y cogeneración de energía eléctrica a operar en el 
Sistema Interconectado Nacional- (Unidad de Planeación Minero Energética - UPME, 2007) modificada en su artículo $4^{\circ}$ y en los anexos 1, 2, y 3 por la Resolución 638 de 6 de diciembre de 2007, para incorporar lo relacionado con los proyectos de generación de energía eléctrica con FNCE se expidió la Resolución 143 de 10 de marzo de 2016.

El artículo 3 de la Resolución 143 establece el procedimiento de registro de proyectos de generación de energía eléctrica a partir de FNCE, para lo cual se debe cumplir con el artículo $4^{\circ}$ de la Resolución 0520 de 2007, es decir, que dependiendo de la fase en que se encuentre el proyecto y conforme a la capacidad de generación (mayores a 1 MW, menores $1 \mathrm{MW}$ ), se debe diligenciar uno u otro formato, los cuales se encuentran como anexos a la resolución.

Así mismo, cuando el proyecto se encuentre en etapa de preinversion o de medición y evaluación del potencial del recurso, se deberá registrar el proyecto de generación correspondiente, utilizando el formato que se emplea para proyectos mayores de $1 \mathrm{MW}$.

Para obtener la certificación de beneficio ambiental por Inversiones en Proyectos de Fuentes No Convencionales de Energía Renovables (FNCER), el Ministerio de Ambiente y Desarrollo Sostenible expidió la Resolución de 1283 del 3 de agosto de 2016, en la cual se define y establecen los requisitos para obtener la certificación, y adicionalmente aclara que conforme al Decreto Ley 3573 de 2011 se creó la Autoridad Nacional de Licencias Ambientales (ANLA) y dentro de sus funciones se encuentra la de otorgar o expedir las licencias, permisos y trámites del Ministerio de Ambiente y Desarrollo Sostenible.
El objeto de la resolución es establecer el procedimiento y los requisitos para obtener la Certificación de Beneficio Ambiental por Nuevos Inversiones en Proyectos de FNCER, certificación necesaria para acceder a los beneficios tributarios de los artículos 11 a 14 de la Ley 1715 de 2014.

En síntesis, el marco normativo es el siguiente: los incentivos fiscales están contenidos en el Capítulo III "Incentivos a la Inversión en Proyectos de Fuentes No Convencionales de Energía" de la Ley 1715 de 2014. Posteriormente, los lineamientos para la aplicación de los incentivos se establecieron a través del Decreto 2143 de 2015; dichos lineamientos se desarrollaron a través de resoluciones expedidas por la UPME-Resoluciones 520 y 638 de 2007, Resoluciones 143 y 045 de 2016- y la ANLA -Resolución 1283 de 2016-.

\section{E. Descripción del proceso de solicitud}

El interesado en acceder a los beneficios de la Ley 1715 de 2014, ya sea una persona natural o una persona jurídica debe cumplir con el siguiente procedimiento.

\section{Registro del proyecto de Generación} y Cogeneración: el primero paso es solicitar el registro del proyecto de generación y cogeneración a operar en el Sistema Interconectado Nacional (SIN), registro que es llevado por la UPME. Para realizar el registro se debe guiar por las resoluciones $520 \mathrm{y}$ 638 de 2007 y 143 de 2016, expedidas por la UPME. Dichas dos resoluciones iniciales contienen el marco general para el registro de los proyectos; la Resolución 143 incorpora los formatos que deben diligenciar para los proyectos de FNCER, la UPME tomará un 
máximo de 30 días calendario para expedir el certificado de registro en cualquiera de sus fases, una vez completada toda la información requerida (Unidad de Planeación Minero Energética - UPME, 2007).

Los formatos se encuentran entre los anexos de la resolución, el primero de los cuales consiste en la información general del proyecto, razón por la cual dicho formato solicita información de identificación de la empresa o persona natural desarrolladora del proyecto, ubicación del proyecto, costos, entre otros. El segundo formato tiene que ver con las características específicas de la Fuente No Convencional de Energía, poder calorífico de la fuente, descripción de los equipos principales.

\section{Certificación que avala la documen-} tación de los equipos, elementos, maquinarias y/o servicios: el siguiente paso es solicitar la certificación que avala la documentación de los equipos, elementos, maquinarias y/o servicios destinados a nuevos proyectos de generación a partir de FNCE. Para tal fin, la UPME expidió la Resolución 045 de 2016, en la cual se establecen los formatos y procedimientos que se deben seguir, la decisión de otorgar o no la certificación que avala la documentación de los equipos se base en la Lista de Bienes y Servicios Excluidos de IVA y Exentos de Gravamen Arancelario (Congreso de la República) que se destinen a nuevas inversiones y preinversiones para la producción y utilización de energía a partir del FNCE, la cual es publicada por la UPME a través del sitio web de la entidad.

El artículo 5 de la citada resolucion indica el procedimiento para solicitar la Certificación de la Documentacion para Obtener el Beneficio de Exclusion del IVA y Exención del Gravamen Arrancelario, para cuyo efecto el solicitante debe diligenciar y entregar en la UPME:

- Formato de presentación para solicitar la Certificación de los beneficios.

- Formato de especificaciones del elemento, equipo, maquinaria y/o servicios.

- En caso de ser persona natural el solicitante, copia del documento de identificación.

- Cuando sea una persona jurídica, Certificado de Existencia y representación legal con fecha de expedición no mayor a un mes.

- Describir la función que cumplen los elementos, equipos, maquinaria y/o servicios que se van a adquirir.

- Catálogos, planos descriptivos o documentos que incluyan especificaciones técnicas.

Esta informacion debe ser entregada en correspondencia de la UPME, a través de una comunicación con asunto "Solicitud de certificacion para incentivos FNCE - Ley 1715 de 2014", acompañada de los archivos digitales de los soportes (Unidad de Planeación Minero Energética, 2016).

La UPME tiene 15 días calendario para expedir la certificación, y en ese mismo tiempo puede solicitar que sea ampliada la información suministrada por el solicitante; el mismo plazo aplica al solicitante para dar respuesta al requerimiento del comité evaluador. A partir de la respuesta a la ampliación, la UPME tiene 15 días para aceptar o rechazar la solicitud, la cual debe ser so- 
portada exponiendo las razones; en otras palabras, se presenta la solicitud a la UPME, entidad que en un plazo de 15 días debe expedir la certificación o solicitar ampliación de la información que acompañó la solicitud, a lo cual el interesado tiene 15 días para entregar la nueva solicitud de información, el comité evaluador tiene 15 días para dar respuesta contados desde el día siguiente a la radicación de la respuesta.

En el caso de que los equipos, elementos y maquinaria nacionales o importados y los servicios ya sean adquiridos dentro o fuera del territorio nacional no se encuentre en la lista, la misma Resolución 045 de 2016 establece el procedimiento para que sea ampliada y/o actualizada la lista a través de los formatos establecidos en el artículo 4; para dar respuesta a la solicitud, la UPME tiene los mismos plazos que aplica para la expedición de la certificación. El procedimiento es similar para la solicitud de aval de los documentos de los equipos y servicios, pero en el formato de presentación se debe diligenciar la casilla de actualización y/o ampliación del listado. El detalle de este procedimiento se encuentra en el artículo 4 de la resolución.

\section{Certificado de Beneficio Ambiental} por Nuevas Inversiones en Proyectos de Generación con FNCER expedido por la ANLA: el tercer paso consiste en obtener el Certificado de Beneficio Ambiental por Nuevas Inversiones en Proyectos de Generación con FNCER expedido por la ANLA; la regulación para esta certificación se encuentra en la Resolución 1283 de 2016; dicha resolución tiene un marco general que define el procedimiento y los requisitos que debe cumplir para acceder a los beneficios; también define unos requisitos específicos dependiendo de si se opta por el beneficio de la Deducción Especial del Impuesto a la Renta y Complementarios y/o la Exclusión del IVA de los artículos 11 y 12 de la Ley 1715 de 2014.

El procedimiento general consiste en radicar ante la ANLA el formato único de solicitud de beneficios tributarios para FNCER, firmado por el representante legal o apoderado del solicitante, acompañado, entre otros documentos, de una descripción detallada de la nueva inversión en proyectos de FNCER y una descripción y cuantificación detallada de los beneficios ambientales asociados al proyecto objeto de la nueva inversión.

Los requisitos específicos dependen de qué beneficio se está solicitando: para la deducción especial del artículo 11 de la Ley 1715 de 2014, se debe entregar adicionalmente el formato de "Especificaciones del Elemento, Equipo, Maquinaria", el concepto de la UPME que avale el proyecto de FNCER y los equipos, elementos, maquinaria, nacional o importados, y declarar bajo juramento que la inversión no se realizara por mandato de una autoridad ambiental.

Para esto, el solicitante deberá entregar a la ANLA el formato único de solicitud de beneficios tributarios para fuentes no convencionales de energías renovables y gestión eficiente de energía, firmado por representante legal o apoderado (Ministerio de Ambiente y Desarrollo Sostenible, 2016), acompañado de los siguientes documentos:

- Copia documento de identidad, si es persona natural.

- Certificado de existencia y representación legal no mayor a un mes cuando se trate de persona jurídica. 
- Poder cuando se haga a través de apoderado.

- Descripción del proyecto en el cual se realizará la inversión.

- Descripción detallada de la nueva inversión en proyectos FNCER, detallando, entre otros aspectos, el objeto y finalidad de la inversión, descripción de las etapas, descripción de la función que realizará cada elemento o equipos a adquirir, ubicación geográfica; cuando se trate de implementación de equipos para proyectos de generación y autogeneración a través de FNCER, se debe señalar la fuente no convencional.

- Descripción y cuantificación detallada de los beneficios ambientales asociados, indicando, entre otros aspectos, el cálculo de la energía media generada en kWh/año, cantidad de $\mathrm{CO}_{2}$ eq que se generaría sin la nueva inversión y la cantidad de $\mathrm{CO}_{2}$ eq que se genera con la nueva inversión.

- Catálogos y planos debidamente firmados por el desarrollador del proyecto y documentos que especifiquen las características técnicas de elementos, equipos y/o maquinaria.

- Cuando el proyecto se encuentre en fase de inversión o de operación, se debe adjuntar copia de la licencia ambiental y en el caso de no requerir de licencia, documento expedido por la autoridad competente que certifique este hecho. Cuando esté en fases de preinversión o inversión pero en estudios técnicos, financieros, económicos y ambientales, no se requiere la licencia ambiental.

Para obtener la certificación de beneficios ambientales para acceder a la deducción especial en el impuesto a la Renta y Complementarios, adicional a los requisitos anteriores, que son de carácter general, también se debe cumplir con los siguientes requisitos específicos:

- Diligenciar el formato de "Especificaciones delElemento, Equipos, Maquinaria” (Formato 1 Anexo a la Resolución 1283 de 2016).

- Concepto de la UPME avalando el proyecto de FNCER y los equipos, elementos y maquinaria, nacionales o importados.

- Declarar bajo la gravedad del juramento que la inversión no es producto de un mandato de una autoridad ambiental.

Cuando se trate de la exclusión del IVA del artículo 12 de la Ley 1715 de 2014, se requiere el formato de "Especificaciones del Elemento, Equipo, Maquinaria” y, si se incluyen servicios, se debe entregar el formato de "Especificaciones de los Servicios". Estos dos formatos deben atender a las listas expedidas por la UPME, y en concordancia con el beneficio de la deducción especial la Certificación de la UPME dando aval al proyecto. Los requisitos de carácter específico son las siguientes:

- Diligenciar el formato de "Especificaciones del Elemento, Equipos, Maquinaria" (Formato 1 Anexo a la Resolución 1283 de 2016), para lo cual se debe contrastar contra la lista expedida por la UPME.

- Cuando se incluyan servicios se deben soportar con el formato "Especificaciones de los Servicios" (Formato 2 Anexo a la Resolución 1283 de 2016), la cual se debe contrastar contra la lista expedida por la UPME. 
- Certificación de la UPME avalando el proyecto de FNCER y los equipos, elementos y maquinaria, nacionales o importados, o la adquisición de servicios.

La ANLA tiene 5 días hábiles para expedir el acto que da inicio al trámite; en los siguientes 10 días hábiles, debe evaluar la información y en ese plazo podrá requerir al solicitante información adicional. Desde la fecha de ejecutoria del inicio del trámite o de entrega de la información adicional, la ANLA tiene 25 días hábiles para expedir o negar la certificación de beneficios ambientales, decisión que debe estar soportada exponiendo las razones.

Quienes reciban la certificación que se obtiene para deducción especial del Impuesto de Renta y Complementarios podrán acceder al beneficio de la depreciación acelerada, es decir, para el beneficio del artículo 14 de la Ley 1715 de 2014, se debe cumplir con los mismos requisitos de la deducción especial.

Para acceder al beneficio de exención del gravamen arancelario aplica el mismo procedimiento de exclusión de IVA, es decir, a quienes se les excluya del IVA automáticamente pueden aplicar a la exención del pago de los derechos arancelarios, pero exclusivamente a las etapas de preinversión e inversión.

El trámite de solicitud de la certificación expedida por la ANLA se debe gestionar a través de la Ventanilla de Trámites Ambientales en Línea (VITAL).

La ANLA tiene la obligación de enviar copia de la certificación a la Subdirección de Gestión de Fiscalización Tributaria o a quien haga sus veces en la DIAN y a la UPME; dicha certificación solo tendrá vigencia de un año.

\section{Conclusiones}

Entre las finalidades de la Ley 1715 de 2017, se debe "estimular la inversión, la investigación y el desarrollo para la producción y utilización de energía a partir de fuentes no convencionales de energía, principalmente aquellas de carácter renovable, a través de mecanismos de carácter tributario, arancelario y contable"; este conjunto de incentivos se encuentran en el Capítulo III de la Ley, pero al hacer la revisión da la percepción de que los beneficios, más que promover la investigación, están dirigidos a la construcción de proyectos de generación con las FNCE. El carácter de los incentivos tributarios (deducción especial, depreciación acelerada, exclusión de IVA y exención de arancel) están vinculados a la compra de maquinaria, elementos y equipos, y a la contratación de servicios relacionados con la construcción de este tipo de activos.

En relación con la deducción especial del $50 \%$ de valor total de las inversiones realizadas en proyectos de generación con FNCE, como está contemplado el beneficio en este momento, al aplicar la deducción especial, este beneficio no se le puede reconocer al accionista y para este caso basta con remitirse al artículo 49 del Estatuto Tributario, a la forma actual de cálculo de los dividendos y participaciones no gravados: la renta adicional que se obtenga por aplicar el beneficio termina gravada para el accionista.

La depreciación acelerada consiste en la posibilidad de tener una tasa anual de de- 
preciación no mayor del 20\%. Este régimen de depreciación acelerada no es un beneficio que sea trasladable a los accionistas: desde la óptica de la empresa que realiza la inversión, un canon mayor de depreciación implica un menor valor del impuesto de renta en el periodo corriente, es decir, si la empresa aplica el canon del $20 \%$, permitido por el artículo 14 de la Ley 1715 de 2014, equivale a depreciar el $100 \%$ del proyecto en un periodo de 5 años, con lo cual en el periodo remanente de la vida útil estimada, la compañía inversora no tiene deducción por depreciación, implicando en un incremento del Impuesto de Renta en esos años. El diferimiento del pago del tributo genera un mayor flujo de caja, el cual tiene un reconocimiento a nivel financiero por el valor del dinero en el tiempo.

Desde la óptica de la persona natural accionista de compañías que realizan inversiones en FNCER, en este tipo de proyectos la mayor deducción por concepto de depreciación fiscal implica un menor Impuesto de Renta corriente en el año gravable que toma el incentivo contable, no representa un incremento en los dividendos que puede percibir inicialmente; esto se debe a un concepto contable que se llama impuesto diferido: al existir diferencias temporarias entre las cifras contables y fiscales, por técnica contable (en este caso NIC 12) se deben constituir los impuestos diferidos, cuya intención es evitar que se anticipen o se retengan utilidades a los accionistas originadas por las diferencias que se presentan entre las cifras contables y las consignadas en la declaración de renta y sobre las cuales existe una expectativa razonable que se van a revertir en el tiempo. En este caso, como la deducción fiscal es mayor al gasto contable, se reconoce un gasto por impuesto diferido, reduciendo la utilidad contable, que es la susceptible de distribuirse.

A nivel operativo, al realizar el proceso de solicitud de beneficios tributarios de la Ley 1715 de 2014, lo primero que se nota es la duplicidad de información y formatos que solicitan la UPME y la ANLA; por ejemplo, los formatos de presentación en ambas entidades son similares y los cambios son menores; así mismo, el tipo de beneficio que se desea acceder, en el caso de la UPME se especifica si es para IVA, arancel o actualización del lista de los equipos y servicios que tiene los beneficios; en cambio, el formato de la ANLA solicita que se defina si el beneficio es para IVA o para Renta.

Lo segundo que se identifica es lo relacionado con la etapa del proyecto: para la UPME es preinversión o inversión, y para la ANLA es preinversión, inversión y operación.

En cuanto a los formatos específicos de los equipos y/o servicios de las dos entidades, las diferencias también son menores, y van encaminados al beneficio al cual se dirige la solicitud; adicionalmente, la ANLA separa la solicitud de beneficios relacionada con los servicios y la maneja en un formato aparte, el cual es simplificado en comparación con el empleado para los bienes.

Al comparar la información complementaria que acompaña los formatos es similar, siendo más amplia y específica la solicitada la ANLA, dado que se debe adjuntar copia de los planos, catálogos, memorias de cálculo de las emisiones y la reducción de las emisiones por el empleo de las FNCE; adjuntar la información dos veces a dos entidades distintas hace que la solicitud se convierta en un reproceso para el contribuyente solicitante. 


\section{Bibliografía}

Betancor, A.; Blanco-Uribe, A.; Brañes, R.; Casas, S.; Johana, D. M.; García Villegas, E. y otros (2002). Lecturas sobre derecho del medio ambiente. Tomo III. Bogotá: Universidad Externado de Colombia.

Bokobo Moiche, S. (2000). Gravámenes e Incentivos Fiscales Ambientales (primera edición). Madrid: A. G. Cuesta, S.A.

Gago Rodríguez, A. y Labandeira Villot, X. (1999). La Reforma Fiscal Verde Teoría y Práctica de los Impuestos Ambientales. Madrid: Ediciones Mundi-Prensa.

García Galludo, M.; Goded Velarde, J. y Suárez Navarro, M. (2001). Energías renovables. Madrid: E.T.S. Ingenieros de Caminos, Canales y Puertos.

Isagén S.A.E.S.P. (2005). Fuentes no convencionales de generación de electricidad (S. Botero Botero, F. Chejne Janna y J. Rodríguez Sanabria, edits.) Bogotá: Impresiones Rojo.

Sarmiento López, A. H. (2010). "Panorama de las fuentes no convencionales de energía”, en Revista Javeriana, 146(761), 813 .

Unidad de Planeación Minero Energética (UPME) (2015). Integración de las energías renovables no convencionales en Colombia. Bogotá: La Imprenta Editores S.A.

Unidad de Planeación Minero Energética (UPME) e Instituto de Hidrología, Meteorología y Estudios Ambientales (IDEAM) (2005). Atlas de Radiación Solar de Colombia. Bogotá, Colombia: IDEAM.

Unidad de Planeación Minero Energética (UPME) e Instituto de Hidrología, Meteorología y Estudios Ambientales (IDEAM) (2006). Atlas de Viento y Energía Eólica en Colombia. Bogotá: IDEAM.

Unidad de Planeación Minero Energética (UPME); Instituto de Hidrología, Meteorología y Estudios Ambientales (IDEAM); Departamento Administrativo de Ciencia, Tecnología e Innovación (COLCIENCIAS) y Universidad Industrial de Santander (UIS) (2009). Atlas del potencial energético de la Biomasa Residual en Colombia. Bogotá: UPME (eds.).

Unidad de Planeación Minero Energética (2007). Plan Energético Nacional (PEN). Ministerio de Minas y Energía. Bogotá: Ministerio de Minas y Energía.

Vega de Kuyper, J. C. y Ramírez Morales, S. (2014). Fuentes de Energía, Renovables y No Renovables. Aplicaciones (1a edición). México: Alfaomega Grupo Editor, S.A. de C.V.

Real Academia de la Lengua Española (2016). http://www.rae.es/.

XM. http://www.xm.com.co. Recuperado de

http://informesanuales.xm.com.co/2016/SitePages/operacion/2-6-Capacidad-efectiva-neta.aspx

\section{Normas}

Leyes

Congreso de la República (2014). Ley 1715 de 2014.

Congreso de la República (2016). Ley 1819 de 2016. 


\section{Decretos}

Ministerio de Minas y Energía (2014). Decreto 2469 de 2014.

Ministerio de Minas y Energía (2015). Decreto 2143 de 2015.

Presidencia de la República (1989). Decreto 628 de 1989.

\section{Resoluciones}

Ministerio de Ambiente y Desarrollo Sostenible (2016). Resolución de 1283 del 3 de agosto de 2016.

Unidad de Planeación Minero Energética (UPME) (2007). Resolución 0520 del 9 de octubre de 2007.

Unidad de Planeación Minero Energética (UPME) (2007). Resolución 0638 del 9 de octubre de 2007.

Unidad de Planeación Minero Energética (UPME) (2016). Resolución 045 del 3 de febrero de 2016.

\section{Jurisprudencia}

Corte Constitucional, Sentencia C-465 de 1993.

Corte Constitucional, Sentencia C-419 de 1995.

Corte Constitucional, Sentencia C-126 de 1998.

Corte Constitucional, Sentencia C-594 de 2010.

Corte Constitucional, Sentencia C-595 de 2010. 